\title{
Acute Limb Ischemia in COVID-19 Patients: A Retrospective Cohort Study
}

Fadhil G Al Amran ${ }^{1}$, Bahaa AL-Madhhachi' ${ }^{1}$, Osamah Obaid Ibrahim², Husam Natheer Naser³, Zaid Alobeid ${ }^{3}$ and Ahmed Muhi Fahad $3^{*}$

\author{
${ }^{1}$ University of Kufa, College of Medicine, Najaf Cardiac Center, Najaf, Iraq \\ ${ }^{2}$ The University of Thi-Qar, Imam Hussein Teaching Hospital, Nasiriya, Iraq \\ ${ }^{3}$ Najaf Cardiac Center, Najaf, Iraq
}

\begin{abstract}
This study aimed to show the incidence, presentation, management, and outcome of acute limb ischemia (ALI) in patients diagnosed with COVID-19 pneumonia, and prove the association between them. A multi-centre retrospective cohort study in the middle and south of Iraq, comparing 400 patients admitted to the isolation hospitals for COVID-19 pneumonia with a matched control group at period from $1^{\text {st }}$ June 2020 to $1^{\text {st }}$ August 2020. A total of 21/400 cases of ALI in COVID-19 pneumonia and only 4/400 cases in the non-COVID-19 group. Relative Risk (Risk Ratio $=5.25,95 \% \mathrm{CI}=1.818-15.157, P=0.001)$. There were 291 (72.7\%) males and 109 (27.2\%) females. The average time from diagnosis of COVID-19 to the onset of ALI was (9.4 \pm 6.4 days). The mean ischemic time was $(22.05 \pm 18.8$ hours $)$ in the COVID-19 group, while it was $(11.75 \pm 8.7$ hours) in the non-COVID-19 group. The mean D-dimer level for the patient with COVID-19 pneumonia without ALI is $(1705 \pm 1256 \mu \mathrm{g} / \mathrm{L})$, while it is $(3730 \pm 2373 \mu \mathrm{g} / \mathrm{L})$ for the patient with COVID-19 pneumonia. The embolectomy done in 14 patients (66.6\%) of the COVID-19 pneumonia group, and it was successful in 10 patients (71.4\%). In comparison, it was done in 3 patients $(75 \%)$ of the control group and was successful in 2 (66.6\%). Amputation was done in 4 patients (19\%) of the COVID-19 pneumonia group, while only one patient (25\%) in the control group had amputation. The association between COVID-19 pneumonia and ALI incidence; the D-dimer level is also associated with ALI in COVID-19 pneumonia cases. The anticoagulants, antiplatelet, vasodilators, and embolectomy had a management success rate.
\end{abstract}

Keywords: acute limb ischemia; COVID-19 pneumonia; D-dimer

\section{INTRODUCTION}

In the past 20 years, the world had witnessed many epidemics, like severe acute respiratory coronavirus syndrome (SARS-CoV), influenza A subtype $\mathrm{H} 1 \mathrm{~N} 1$, and Middle East coronavirus respiratory syndrome (MERS-CoV) (Cascella et al., 2020). The COVID-19 is highly contagious and spread very fast across the world, and WHO declared the pandemic on $30^{\text {th }}$ January 2020 (Chen et al., 2020). COVID-19 has a broad spectrum of presentation ranged from asymptomatic to severe respiratory distress, multiple organ dysfunction, and death (Yazan et al., 2020). Data suggest that COVID-19 is associated with a hypercoagulable state and elevated D-dimer level
(Yazan et al., 2020). Acute limb ischemia (ALI) can occur due to concomitant cardiovascular or cerebrovascular disease and ischemia-reperfusion injury and may be ended with amputation, unless appropriate treatment is initiated (Norgren et al., 2007). It is associated with a mortality rate of (15-20\%) due to concomitant cardiovascular or cerebrovascular disease and ischemia-reperfusion injury (Norgren et al., 2007). The aetiology of ALI classified into embolism (46\%) and thrombosis (54\%), and atrial fibrillation account for most cases of embolism (Norgren et al., 2007). The femoral artery is the most common site where embolus is lodged (Norgren et al., 2007). The 
thrombosis can occur from plaque breakdown, circulatory failure, or a hypercoagulable state and include thrombotic occlusion of stents and bypass grafts (Howard et. al., 2015; Creager et al., 2012).

D-dimer is the end product of the degradation of fibrin, and it is a marker of the activated hemostatic system (Pagana et al., 2019). The level of D-dimer increase in thrombosis, pregnancy, inflammation, malignancy, trauma, postsurgical treatment, liver disease, and heart disease (Spring et al., 2014). The D-dimer level is elevation is associated with increased mortality in COVID-19 patients (Zhang et al., 2020).

\section{MATERIALS AND METHOD}

\section{Study design and setting}

This is a multi-centre retrospective cohort study done in middle and south of Iraq comparing 400 COVID-19 patients admitted to the infectious isolation hospitals with another 400 non-COVID-19 patients admitted to other infectious isolation hospitals in the same period from $1^{\text {st }}$ June to $1^{\text {st }}$ August 2020.

\section{Diagnosis}

The ALI was either diagnosed before or after the admission by clinical assessment and duplex scan. According to WHO, COVID-19 infection was confirmed by positive real-time reverse transcription-PCR (RT-PCR) of nasal and pharyngeal swabs or was determined by typical findings on chest CT scan when laboratory testing was inconclusive.

\section{Intervention}

An anticoagulant in the form of enoxaparin 4000 IU once daily was routinely given to all hospitalized patients with COVID-19 pneumonia even if they were not bedridden, whereas it is only given to bedridden in the control group.

\section{Data collection}

We recorded demographic data, co-morbidities, laboratory tests, the anatomical location of the occlusion, the ischemic time, the intervention, complications, and the time of onset of limb ischemia from the diagnosis of COVID-19 pneumonia.

\section{Ethical approval and patients consent}

Written informed consent was obtained from the patients or the parents/guardians of minors for those below the age of 18 years for participating in this study, and was conducted according to the ethical standards established by the 1964 Declaration of Helsinki. The Medical Ethical Committee of
Kufa University and Najaf Cardiac Center approved this study (code:2020NCC172).

\section{Statistical analysis}

We use mean and standard deviation to represent the data while describing variables presented using their numbers and parentage. Relative risk ratio used to estimated risk. Two-sided paired t-test for variables was used. SPSS version 22 (Chicago, US) was used for data entry and analysis. P-value was considered significant if $<0.05$.

\section{RESULT AND DISCUSSION}

21/400 (5.25\%) cases of ALI in COVID-19 pneumonia and only 4/400 (1\%) cases in non-COVID-19 patients. Relative Risk (Risk Ratio) of patients with COVID-19 reliable to develop ALI was 5.25 (95\%CI=1.818-15.157, $P=0.001)$. The

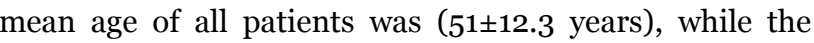
mean age of patients with ALI was (54.1 \pm 11.1 years).

The average time from diagnosis of COVID-19 to onset of ALI was (9.4 \pm 6.4 days) except for one individual, who had ALI as the presenting complaint.

The mean ischemic time was $(22.05 \pm 18.8$ hours $)$, ranging from 3 to 72 hours in the COVID-19 group, while it was (11.75 \pm 8.7 hours) range from 5 to 24 hours in the nonCOVID-19 group, $(P=0.000)$. Three cases of COVID-19 pneumonia ALI group had associated with deep vein thrombosis (14.2\%), whereas this was not found in nonCOVID-19 group.

The mean D-dimer level for the patient with COVID-19 pneumonia without ALI is $(1705 \pm 1256 \mu \mathrm{g} / \mathrm{L})$, while it was $(3730 \pm 2373 \mu \mathrm{g} / \mathrm{L})$ for the patient with COVID-19 pneumonia and ALI $(P=0.002)$. The commonest anatomical arterial occlusion location is in the femoral, popliteal, and tibial arteries (as shown in Figure 1). Embolectomy was done in 14 patients (66.6\%) of the COVID-19 pneumonia group. It was successful in 10 patients (71.4\%). At the same time, it was done in 3 patients (75\%) of the non-COVID-19 and was successful in 2 (66.6\%), $(P=0.36)$.

Amputation was done in 4 patients (19\%) of COVID-19 pneumonia, while it has done in one patient (25\%) in the non-COVID-19 group, $(P=0.4)$. One patient with posterior tibial artery thrombosis had been treated successfully with catheter-directed thrombolysis (as shown in Figure 2). 


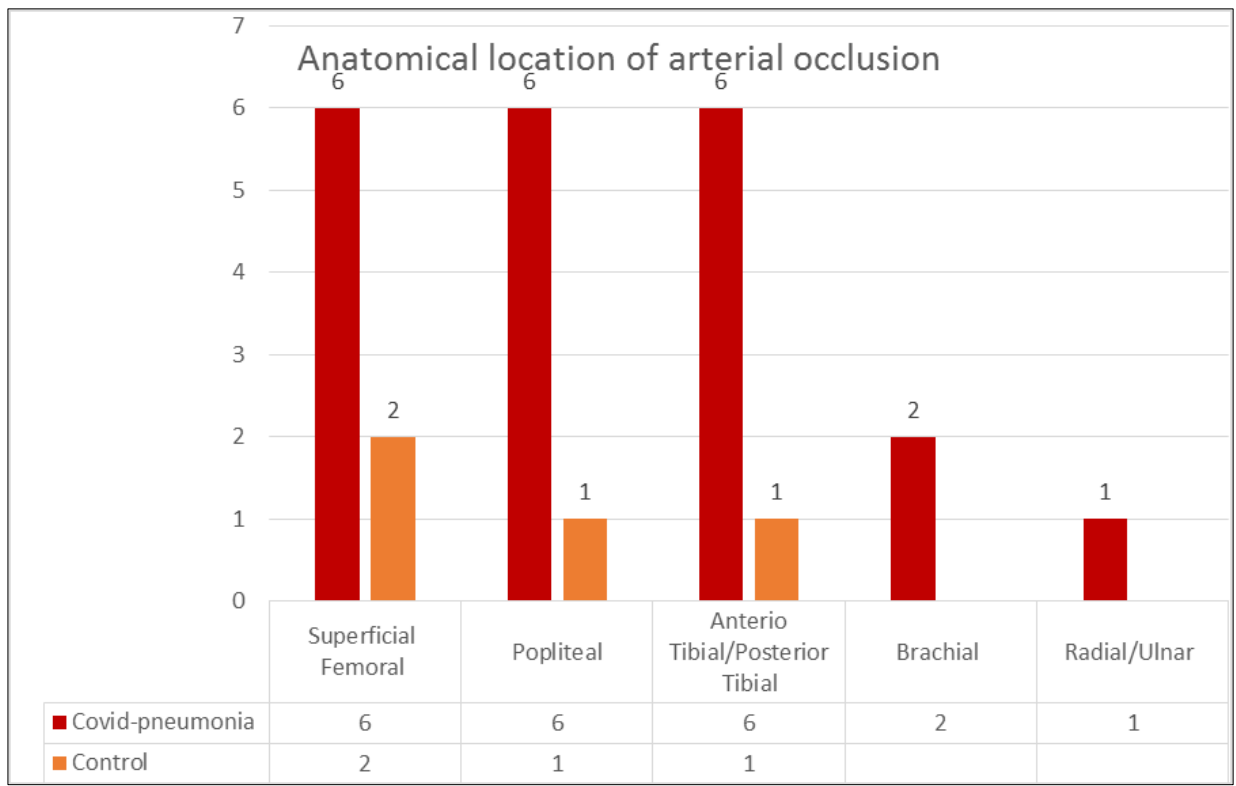

Figure 1. The anatomical location of arterial occlusion

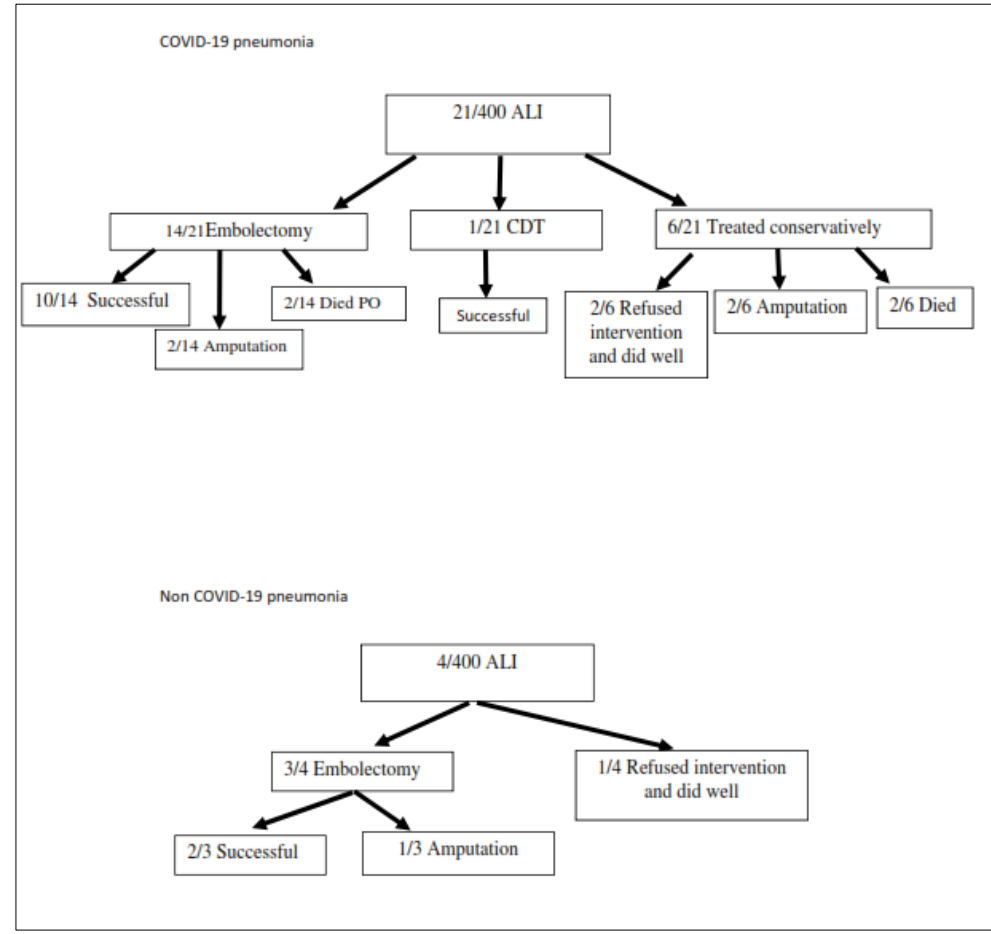

Figure 2. The flow chart of the two arms of the study

Italian team from the Lombardy region reported in April 2020 a significantly increased ALI incidence during the COVID-19 pandemic (Bellosta et al., 2020). They observed a lower success rate of revascularization than expected, which may be due to the hypercoagulable state induced by the virus, and they recommended prolonged systemic heparin use (Bellosta et al., 2020).

In Iraq, ALI's first case report in COVID-19 was published in
July 2020 (Fahad et al., 2020). An exponential increase in the number of COVID-19 cases, associated with an increased incidence of ALI cases. The younger patients than what was seen in Italian study due to the demography difference between the two countries as Italy is the sixth country by life expectancy while Iraq is $130^{\text {th }}$ (www.worldometers.info.2020).

The mean ischemic time is that the COVID-19 
pneumonia group was significantly higher than the nonCOVID-19, which can be explained by the overwhelming of the already collapsed health system. The non-COVID-19 group is still higher than the ischemic time in Italy COVID-19 hospital, where it does not exceed 3 hours (Bellosta et al., 2020).

High D-dimer level is associated with acute thrombotic events (Chiu \& Huang, 2009), and D-dimer level is already known to increase in COVID-19 patients and is associated with increased mortality (Zhang et al., 2020). Here, the conclusion is that a higher D-dimer level is significantly associated with ALI in COVID-19 patients. The presence of associated DVT in the COVID-19 pneumonia group also support the hypercoagulable state theory as the cause of limb ischemia.

We did not do embolectomy either because of late presentation with a gangrenous limb or patient refusal. Our intervention rate is lower than seen in other studies due to longer ischemic time in our patients, but the success rate is comparable (Bellosta et al., 2020).

Our study's mortality rate was significantly lower than the Italian series, which we believe due to younger people in our research, and COVID-19 is well-known to kill older people (Zhou et al., 2020).

\section{CONCLUSION}

There is an association between COVID-19 pneumonia and the incidence of ALI. D-dimer level is also associated with ALI in COVID-19 pneumonia cases. Anticoagulants, antiplatelet, vasodilators, and embolectomy had a success rate in management.

\section{REFERENCES}

Bellosta, R, Luzzani, L, Natalini, G, Pegorer, MA, Attisani, L, Cossu, LG, Ferrandina, C, Fossati, A, Conti, E, Bush, RL \& Piffaretti, G 2020, 'Acute limb ischemia in patients with COVID-19 pneumonia', Journal of Vascular Surgery, vol. 72, no. 6, pp. 1864-1872.

Cascella, M, Rajnik, M \& Cuomo, A 2020, Features, evaluation, and treatment of coronavirus, StatPearls Publishing, < https://www.ncbi.nlm.nih.gov/books/NBK554776/>.

Chen, WH, Strych, U, Hotez, PJ \& Bottazzi, ME 2020, 'The SARS-CoV-2 vaccine pipeline: an overview', Current Tropical Medicine Reports, pp. 1-4.

Chiu, Y \& Huang, M 2009, 'D-Dimer in patients with suspected acute mesenteric ischemia', The American Journal of Emergency Medicine, vol. 27, no. 8, pp. 975-979.

Creager, MA, Kaufman, JA \& Conte, MS 2012, 'Clinical practice.: acute limb ischemia', New England Journal of Medicine, vol. 366, no. 23, pp. 2198-2206.

Fahad, AM, Mohammad, AA, Al-Khalidi, HA, Lazim, QJ, Hussein, FI \& Alshewered, AS 2020, 'Case report: COVID-19 in a female patient who presented with acute lower limb ischemia', F100oResearch, vol. 9, no. 778, pp. 778.

Howard, DP, Banerjee, A, Fairhead, JF, Hands, L, Silver, LE \& Rothwell, PM 2015, 'Population-based study of incidence, risk factors, outcome, and prognosis of ischemic peripheral arterial events: implications for prevention', Circulation, vol. 132, no. 19, pp. $1805^{-1815}$

\section{Coronavirus}

update

2021 ,

https://www.worldometers.info/2020.

Norgren, Lars, William, R, Hiatt, John, A, Dormandy, Mark, R, Nehler, Kenneth, A, Harris \& F, Gerry, R, Fowkes 2007, 'Inter-society consensus for the management of peripheral arterial disease (TASC II)', Journal of Vascular Surgery, vol. 45, no. 1, pp. S5S67.

Pagana, KD, Pagana, TJ \& Pagana, TN 2019, Mosby's diagnostic and laboratory test reference, 14th edn, Elsevier, St. Louis, MO, US.

Spring, JL, Winkler, A \& Levy, JH 2014, 'The influence of various patient characteristics on $\mathrm{D}$-dimer concentration in critically ill patients and its role as a prognostic indicator in the intensive care unit setting, Clinics in Laboratory Medicine, vol. 34, no. 3, pp. 675-686.

Yazan, M, Diamond, A \& Kapoor, S 2020, 'The hypercoagulable state in COVID-19: incidence, pathophysiology, and management', Thrombosis Research, vol. 194, pp. 101-115.

Zhang, L, Yan, X \& Fan, Q 2020, 'D-dimer levels on admission to predict in-hospital mortality in patients with COVID-19', Journal of Thrombosis and Hemostasis, vol. 18, no. 6, pp. 1324-1329.

Zhou, F, Yu, T \& Du, R 2020, 'Clinical course and risk factors for mortality of adult inpatients with COVID-19 
in Wuhan, China: a retrospective cohort study', The Lancet, vol. 395, no. 10229, pp. 1054-1062. 